peritonitis. He, however, considers the extreme anxiety and distress of the patient's countenance as more pathognomonic of the occurrence than the vomiting or state of the pulse.

\section{LONDON MEDICAL SOCIETY.}

SPONTANEOUS PERFORATION OF THE STOMACH.

Dr. Clutterauck communicated the following case from Dr. J. B. Thompson :-

Maria Martin, aged 26, unmarried, of nervous temperament, previously enjoying good health, and of very active habits, had never complained of any gastric derangement. When in the act of putting on her stays, she was suddenly seized with an acute pain in the region of the epigastrium, at eight o'clock in the morning, and the pain continued to increase hourly. Dr. Thompson visited her at twelve o'clock at noon, and found her then labouring under the most intense and seemingly excruciating pain, which she, from her own description, said was confined to a particular part of the stomach, about an inch and a half to the left of the xyphoid cartilage. Leeches were at once applied to this part, and cupping glasses afterwards, and the bleeding was encouraged by stupes and poultices; she was then ordered into a hip bath, and allowed to remain in it for twenty minutes, when she was removed to bed and got an active aperient draught. These remedies apparently afforded some very considerable relief, and the draught had operated twice during the day. She had some undisturbed steep; but the pain again returned at eleven o'clock at night, and seemed to be considerably aggravated, and to occupy the great of the abdomen. She was again visited at two o'cluck, a.m., next morning, when she was found evidently rapidly sinking, and in a semi-comatose state. Dr. Thompson was then only for the first time satisfied that the patient's case was one of spontaneous rupture of the stomach, and that the diffuse peritoneal inflammation then present was owing to the extravasation of the fluid contents of the organ into the peritoneal sac. She gradually got worse, and died in about thirty-five hours from the commencement of her attack.

There was some difficulty in procuring a post-mortem examination. However, the friends were prevailed upon, and she was examined twenty-two hours after death. In the outward appearance of the stomach there was nothing to attract notice, but on opening it there was general inflammation of the surface, and on closely examining its parietes, there was found in the lesser curvature an oval opening in the mucous mem brane; the submucous coat was also similarly perforated, but the opening in the serous coat was not observable at first sight. It was only to be noticed (unless very minutely examined) by passing a probe from the opening in the mucous coat.

It was an oblique slit, not exactly corresponding to the opening in the inner coats; there was nearly a pint of fluid contents of the stomach in the abdominal cavity, and the peritoneum presented appearances of general inflammation. A further examination was not permitted; but the patient seemed in every other respect to have been in good general health.
OBSERVATIONS.

The foregoing is an instance of a very peculiar form of disease which seems to be confined to a point, and terminates in ulceration. The duration of the disease varies from eight to twelve or thirty-six hours; we seldom have an opportunity of seeing the stomach in the early stage of this affection; some are of opinion that it does not prove fatal during the early or inflammatory stage, as would appear to have been the case in the foregoing instance; the ulcer itself does not often destroy life, but it permits the fluid contents of the stomach to pass into the peritoneum, and the resulting peritonitis proves fatal.

The ulcers in these cases are generally of various sizes, from that of a sixpence, as was the case in this instance, to that of a half-a-crown piece. In the more chronic cases, the ulcers are likely to be larger and better defined about the margins. They invariably are oval-shaped or somewhat rounded, particularly in the mucous coat, and in recent cases seem as if they were punched out. The ulceration is mostly noticed in or about the lesser curvature of the stomach, on its posterior inferior surface. There is no reason to believe that the disease is of a cancerous nature. The appearance of the ulcer and its general history ought, in the opinion of the author, to be a sufficient proof that it has its origin in the mucous coat of the stomach, and not, as is invariably the case, in scirrhous affections in the submucous tissue. Besides, we have more of the mucous coat destroyed in these cases than of any other membrane or tissue.

The symptoms of the disease are at first rather obscure. In many cases the genera health appears good, and often no complaint is made till some short time before death, when a most painful and violent train of symptoms present themselves. It would also appear that the nervous and muscular coats are engaged at the same time with the mucous coat, and that it is only when the serous coat becomes engaged that those acute symptoms generally exhibit themselves. Then, either the peritoneal coat gives way, the contents of the stomach are extravasated, and fatal peritonitis ensues, or a degree of partial inflammation may be produced in a small portion of the peritoneum, extended to, but not yet finally destroyed; lymph is thrown out, adhesion takes place between that particular part and some adjoining viscus, and in this manner life may be preserved for the time. Beclard's case was a remarkable instance of this nature ; he died of disease of the brain, and a cicatrix was discovered near the lesser curvature of the stomach, half an inch from the cardiac orifice; it was about the size of a shilling; the surface was depressed and traversed by bands, one of which passed across the centre and divided it into two lacunæ, the base of which rested on the peritoneal coat. Recamier mentions a case in which the pancreas formed the base of a cicatrised ulcer of the stomach; the patient had died of another complaint. Cruveilhier and others do not think such cicatrices are of very rare occurrence.

Usually, however, the ulcer in these cases, destroys life, and in one of three ways :-First, by gradually exhausting the patient's strength, by pain, vomiting, \&c., frequently aided by disease of the intestines, liver, spleen, or pancreas, with perhaps occasional 
dropsy; secondly, by hæmorrhage, which is either the result of slow and often repeated oozings of blood from the ulcer, or sudden, from a large ressel which the ulcer eventually opens; thirdly, by the more quick and violent effects which follow perforation of the stomach, and the consequent extravasation of its contents into the peritoneum. In these suddenly fatal cases, the patients may be taken off in from six or twelve hours before peritoneal inflammation has fully set in, and simply from the shock which the nervous system has sustained, and from which it cannot afterwards rally. We see this occurring daily in cases of severe or extensive scalds or burns, and other serious lesions or injuries; or a violent peritonitis sets in, and destroys the patient in from twenty-four to thirty-six hours.

The diagnosis of ulceration is most difficult; indeed, the author is not aware that we as yet possess any pathognomonic symptom whereby we may with certainty distinguish this disease from other affectionssuch as gastrodynia, scirrhus, functional dyspepsia, chronic ulceration, or chronic inflammation. There is mostly vomiting and irritability of the stomach; but it has been seen without these. There is often pyrosis, which also is often absent. In some cases there is a wasting of the flesh, but in others the embon point is retained. It would appear that persons may remain in apparently perfect health up to the moment at which the ulcer gives way. Dr. Abercrombie gives a case of this kind; and another case is recorded, in one of the Dublin journals, of a young lady who went to church in excellent health, had never complained of any gastric disturbance, but was suddenty, while in church, seized with the symptoms of effusion into the peritoneal sac, and expired in thirty-six hours. The post-mortem examination in this case showed a perforating ulcer with effusion and peritonitis.

A circumscribed inflammation, which is, perhaps, more dangerous than a diffused one, because more likely to be followed by ulceration, may only be attended by slight dyspeptic symptoms, or may even escape notice altogether, until the ulcer has perforated the stomach, and fatal peritonitis ensues. In the more chronic forms of these affections, the mucous membrane seems thickened; its papillæ are very considerably developed; the degree of redness varies from a dusky brown to a somewhat livid or leaden hue; the latter is often seen on detached patches, where it would appear that ulceration would at some future period take place. It is worthy of notice, also, that these cases are more frequently met with in the female than in the male, generally between the ages of eighteen and thirty years, and mostly in unmarried women who were not apparently labouring under any organic or functional derangement. It would be interesting to enquire whether the gastric juice possesses any more active chemical properties at this period, and in this class, than at any other time, or under different circumstances. It is also very remarkable, that all these ruptures or perforations are to be found in or about the lesser curvature. In the few cases which have come under the notice of the author, he has never been able to perceive the thickened and clevated edges that some 'gentlemen speak of; he presumes these must be very chronic cases, and not at all such as those here alluded to.
In all chronic cases of this description there is gene. rally a long and tedious illness, accompanied by all the varied and anomolous distressing symptoms of indigestion, constipation, and considerable hepatic derangement.

An interesting discussion followed the reading of this paper, after which the society adjourned.

\section{MEDICAL SOCIETY OF PARIS.}

SOLUTION OF URINARY CALCULY.

At a recent meeting of this society $M$. Petit related the following interesting case, illustrative of the action of Vichy water on urinary calculi.

The patient, a man named Jacob, had been treated during the season of 1839 , by a course of Vichy waters, but had not received much benefit from them, having neglected to follow the directions given to him. On the 10th of June, 1840, he was examined by MM. Civiale, Blandin, and Berard, who frequently seized the stone between the branches of the instrument, and discovered its diameters to be thirteen, fourteen, and fifteen lines.

The man arrived at Vichy on the 2lst June, and commenced his treatment on the 23rd; this time he followed punctually every direction. The waters and baths were administered in the usual manner, and, in addition, the mineral waters were injected into his bladder, by means of a double syringe, two or three times a day. The dose of the waters taken by the mouth was from twelve to fifteen and often twentyglasses daily.

This treatment was borne without any inconvenience, and the other attendants at the spa took care that it was not interrupted for a single day. Towards the commencement of August the patient began to pass some fragments of calculi; several of these were lost, because he was often compelled to make water when away from home. About the 10th of September the discharge of calculous fragments ceased; the patient was now free from pain and the sensation of a foreign body, and made water freely. On the 18 th he left Vichy, and on the 28th November he was examined by MM. Civiale, Blandin, and Berard, who decided that the bladder was free from stone. The patient now enjoys perfect health, and is completely free from all symptoms of catarrh of the bladder.

This is the only case in which the complete removal of the urinary calculus by the Vichy waters has been clearly demonstrated. M. Petit thinks that the calculus was destroyed rather by disintegration of its particles than by solution; he also remarks that even this case, striking as it may appear to be, leaves some doubt upon his mind, because the patient had undergone a lithotriptic sitting at the hospital Beaujon before he commenced the use of the Vichy waters. Finally M. Petit concludes that the waters of Vichy chiefly act on the mucus which unites the particles of calculi together, and in this he coincides with the opinion of M. Pelouse, which we published in our last number. 